\title{
MODIFIED LAND SUITABILITY EVALUATION MODEL FOR IRRIGATED AGRICULTURE
}

\author{
I. F. RASHAD \\ Soils, Water and Environment Research Institute, ARC, Giza, Egypt.
}

(Manuscript received $2^{\text {nd }}$ August 2016 )

\begin{abstract}
$\mathrm{T}$ he proposed modification of soil rating was performed to study the comparison between different methods of land evaluation for the irrigated arid and semi-arid regions. Therefore, twenty-two soil profiles were chosen to represent some soils of the main geomorphic units within the Nile Delta and its desert fringes in Egypt. These soil profiles were assessed to identify the suitability classes by using the parametric soil rating of Storie and Sys systems as well as the descriptive FAO and USBR schemes. Data revealed the major differences in suitability classes with respect to their either descriptive or parametric system. The modified soil rating model depends mainly on soil characteristics itself. The principle of land classification in arid and semi-arid regions, the concepts of FAO Framework, Storie and Sys methods, as well as the guidelines for soil profile description and the criteria of soil taxonomic units, were all take into consideration. The soil rating chart includes the more stable characteristics (factor a), the relatively less stable characteristics (factor b); surface characteristics (factor $\mathrm{c}$ ), and the miscellaneous (factor $\mathrm{x}$ ) with respect to Suitability index values of $(\mathrm{Si})$ is calculated as:

$\mathrm{Si}=$ Factor (a) $\mathrm{x}$ factor (b) / $100 \mathrm{x}$ factor (c) / $100 \mathrm{x}$ factor (x) /100 However;

S1 $(\mathrm{Si} \geq 80)$ Highly Suitable $\quad$ S4 $(20-39)$ Marginally Suitable

S2 $(60-79)$ Suitable SC $(10-19)$ Conditionally Suitable S3 $(40$ - 59) Moderately Suitable N $(\mathrm{Si}<10)$ Unsuitable

By using the modified rating, the obtained results would become more reliable application and qualitative evaluation of either current or potential suitability classes pertinent the preproject, reconnaissance and even detailed phase of soil survey.
\end{abstract}

\section{INTRODUCTION}

Land use is a set of biological and technological human activities, engaged for economic and social purposes. These activities are directed towards the management and improvement of land resources. Land resources as such are phenomena of nature which are described in strictly scientific terms; they give no indication themselves of how they could or should be used. Land qualities or ecological land conditions are used as a mean for indicating the direct relations between the land as an ecological complex and the biological and technological activities of land use (Vink, 1975). 
For the purpose of judging "land suitability", both for land use and land improvement, a systematic land evaluation system is necessary. Land evaluation is the process of collating and interpreting basic inventories of soil, vegetation, climate, and other aspects of land partly because insufficient quantitative information is available, and partly in order to identify and make a first comparison of promising land use alternatives in simple socio-economic terms (Brinkman and Smyth, 1973). Therefore, land evaluation is an essential tool in land use planning as it is assigned the indispensable task of translating the data on land resources into terms and categories which can be understood and used by all those concerned with land management, land improvement and land development. The qualitative classification is one in which relative suitability is expressed in qualitative terms, without precise calculation of cost and returns. The categories are based mainly on the physical productive potential of the land, with economics only present as a background.

About one-third of the world's land surface suffers from a moisture deficiency which presents a major constraint to agricultural development (Zonn, 1977). Any significant increase in agricultural output from arid and semi-arid regions is dependent upon new irrigation schemes. Such projects are highly expensive and necessitate very careful planning to ensure ultimate economic and social success. A general subjective system of land evaluation for irrigated agriculture has been elaborated by the United States Bureau of Reclamation (USBR, 1953). This system has been applied in many other countries, often after certain modification, but none of these systems has been universally accepted. In view of this, FAO has prepared a manual entitled "Framework for Land Evaluation" (FAO, 1976). This manual which, intended to have world-wide application, is based on the concepts and procedures of land evaluation that have evolved during FAO-assisted development projects.

Land evaluation which is numerically calculated avoids subjective assessments. As described by Beek (1978), parametric methods involve the selection of soil properties which are evaluated and given numerical scores. These scores are subjected to mathematical formulas mostly by multiplied together, so that an overall index of suitability or performance is obtained. An early index was proposed by Clarke (1951) and summarized by Smith and Atkinson (1975). Another widely quoted early 
methods are by Storie (1954), Olsen (1974), Vink (1975) and Storie (1978). The index is obtained by multiplying scores for selected variable and the results have been applied in many studies, often after some modifications for local conditions. Also, Bowser and Moss (1950) trace the development of soil rating methods relevant to irrigation, the system proposed by Sys and Verheye (1974) gives greater emphasis to soil chemical properties suggested by Bowser and Moss (1950). They state that the suitability of soils for irrigation in arid and semi-arid area is mainly influenced by seven factors, viz texture, soil depth, calcium carbonate content, gypsum status, salinity and alkalinity content, soil drainage and slope. However, with the guidance of FAO Framework for Land Evaluation (FAO, 1976), the parametric simple approach proposed by Sys and Verheye (1978), modified and improved by Sys et. al. (1991 and 1993) are widely used to identify suitability categories. Generally, these methods are mainly depending upon the same principles but with some modifications. It is noticed that these methods have differences in their suitability index values.

This work aims to compare the obtained results from application of the most common used methods in some soils of Egypt as arid region, within the proposed modifications of some soil ratings.

\section{MATERIALS AND METHODS}

\section{1-Soil data:}

According to El-Nahal et. al. (1977) and Veenenbos (1963), twenty-two investigated sites were chosen to represent some soils in the main geomorphic units in the Nile Delta and its desert fringes (Fig. 1). The soil profiles in these sites were morphologically described according to the methods undertaken by both FAO (1990) and USDA (1993). Representative soil samples were collected from the different profile layers. for physical and chemical analyses. 


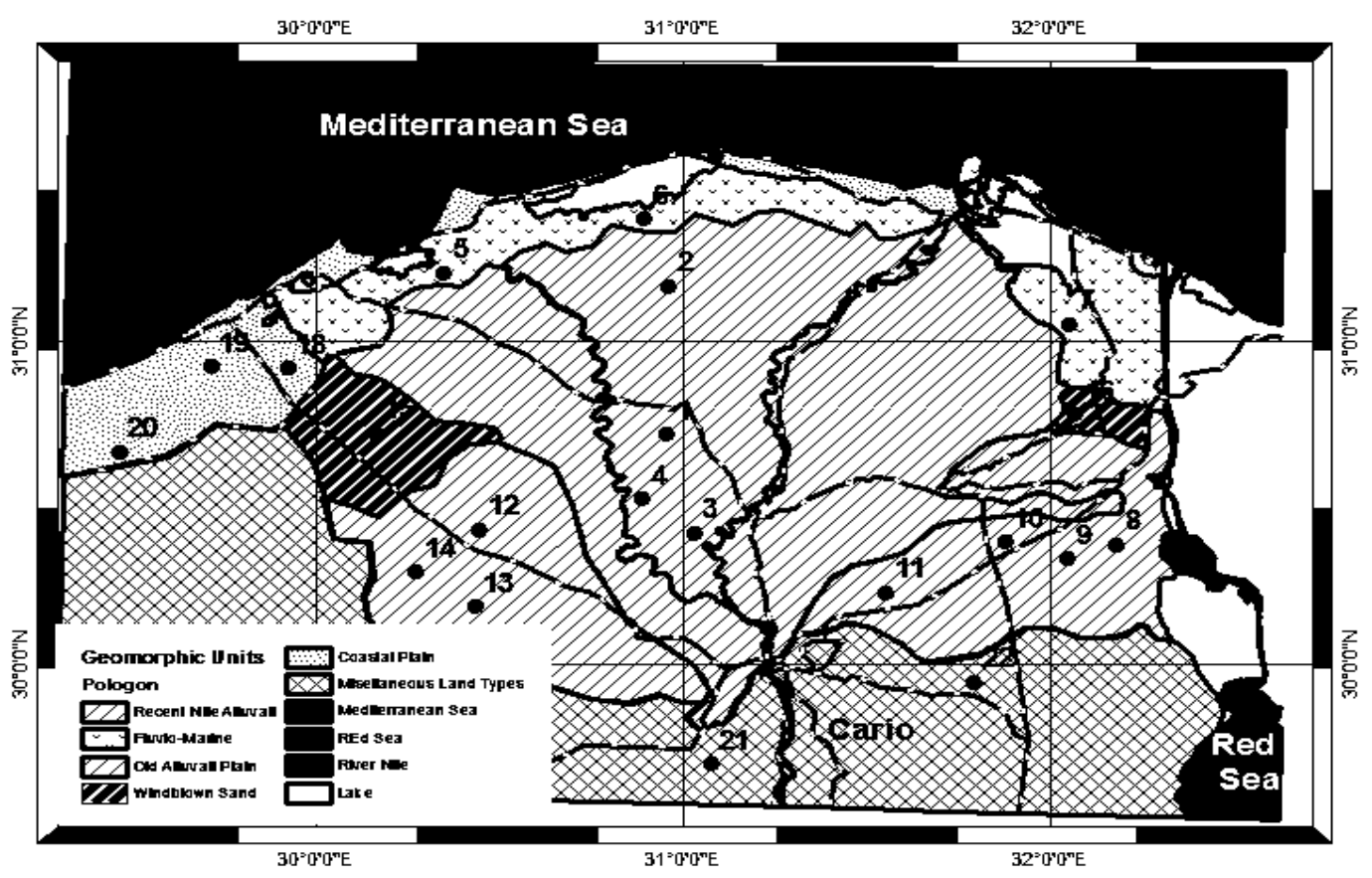

Fig. 1. Main geomorphic units and location of the investigated soil profiles.

According to USDA (2004), the fine earth of soil samples were analyzed for particle size distribution by pipette method; soil reaction $(\mathrm{pH})$ in the saturated soil paste and soil salinity as Electrical Conductivity (ECe) of the saturated soil extract; Cation Exchange Capacity (CEC) and Exchangeable Sodium Percent (ESP) using ammonium chloride solution of $\mathrm{pH}$ 8.5; Organic Matter content (OM) using the modified method of Walkley and Black; $\mathrm{CaCO}_{3} \%$ by using the Collin's Calcimeter; and gypsum content by precipitation with acetone. The soil profiles were classified to the family level according to the Soil Taxonomy System (USDA, 2014).

\section{2-Land suitability evaluation methods:}

Land suitability evaluation for surface irrigation was achieved according to the following systems:

\section{Descriptive methods:}

- Suitability evaluation regarding to individual soil characteristics (FAO, 1979).

- Suitability evaluation regarding to land qualities (Sys et. al., 1991).

- The general system of USBR modified by Griffiths (1975).

- USBR system correlated with the FAO land classification (FAO, 1979).

\section{Parametric methods:}

- Storie method modified by Nelson (1963).

- Storie Index Soil Rating (1978).

- Sys and Verheye (1974 and 1978) and Sys et al. (1991). 


\section{RESULTS AND DISCUSSION}

\section{Soil characteristics:}

The differences in soils are closely associated with variation in its origin that influences the nature of soils, as well as the effect of man through land reclamation. The investigated soils, which extensively mantled with Pleistocene and Holocene epochs, include examples of soils derived from recent Nile alluvial Delta as old cultivated land of Egypt; the old and recent reclaimed soils located in the north along the sea coast and northern lakes as Fluvio-Marine soils; the desert fringes on both sides of the Delta as old alluvial soils; the soils derived from active Aeolian processes as windblown sand soils; the coastal plain soils as depressed Sabkhas and MarineLacustrine plain; and Miscellaneous land types. The morphological features, analytical data, and soil taxonomic units characterized the chosen soil profiles were illustrated in Tables 1, 2 and 3, respectively.

\section{Suitability evaluation for irrigated agriculture:}

The obtained suitability classes by using the investigated methods, which are made on qualitative bases under surface irrigation, are shown in Table (4). Generally, the results indicate different suitability classes between numbers of the examined soil profiles and can be discussed as follows:

\section{a- Descriptive methods:}

- The USBR scheme (Griffiths, 1975) is not only concerned with soil conditions but with all the factors which influence the ultimate financial returns once an irrigation schemes is installed. Accordingly, only 3 out of 9 sites that represent the cultivated soil under surface irrigation indicate the same suitability class compared with FAO (1979) method.

- Land suitability regarding to individual soil characteristics (FAO, 1979) indicates different classes in $50 \%$ of the examined sites if compared with the land qualities assessment (Sys et al., 1991). There is no clear trend of the more or less grade between the two methods. 
Table 1. Morphological features of the investigated soils.

\begin{tabular}{|c|c|c|c|c|c|c|}
\hline $\begin{array}{c}\text { Main } \\
\text { Geomorphic } \\
\text { unit }\end{array}$ & $\begin{array}{l}\text { Recent Nile alluvial } \\
\text { soils } \\
\text { (Nile Delta) }\end{array}$ & $\begin{array}{l}\text { Fluvio-Marine } \\
\text { soils }\end{array}$ & Old alluvial plain soils & $\begin{array}{l}\text { Windblown } \\
\text { sand soils }\end{array}$ & Coastal plain soils & $\begin{array}{c}\text { Miscellaneous land } \\
\text { types }\end{array}$ \\
\hline Profile No. & $1,2,3,4$ & $5,6,7$ & $8,9,10,11,12,13,14$ & 15,16 & $17,18,19,20$ & 21,22 \\
\hline $\begin{array}{l}\text { Landform } \\
\text { elements }\end{array}$ & $\begin{array}{l}\text { Almost flat floodplain ( } 1 \text {, } \\
2) \text { or levee }(3,4) ; 5 \text { to } \\
14 \mathrm{~m} \text {. above sea level } \\
\text { (a.s.l) }\end{array}$ & $\begin{array}{l}\text { Locally lagoons ; < } \\
1.0 \mathrm{~m} \text { (a.s.l) }\end{array}$ & $\begin{array}{l}\text { Almost flat to undulating } \\
\text { old deltaic plain }(8), \text { old } \\
\text { river terraces }(9,10,11, \\
12) \text { valley floor }(13), \\
\text { piedmont (14); } 30 \text { to } 120 \\
\mathrm{~m} \text { (a.s.l) }\end{array}$ & $\begin{array}{l}\text { Aeolian low sand } \\
\text { dunes; } 18 \text { to } 30 \\
\text { m. (a.s.l) }\end{array}$ & $\begin{array}{l}\text { Depressed sabkha (17) } \\
<1.0 \mathrm{~m} \text { (a.s.l); almost flat } \\
\text { to gently undulating } \\
\text { marine-lacustrine plain } \\
(18,19,20), 20 \text { to } 28 \mathrm{~m} \text {. } \\
(\text { a.s.l) }\end{array}$ & $\begin{array}{l}\text { Almost flat to gently } \\
\text { undulated. denuded } \\
\text { rock-land (21) or } \\
\text { plateau remnants (22); } \\
150 \text { to } 170 \mathrm{~m} \text {. (a.s.l) }\end{array}$ \\
\hline $\begin{array}{c}\text { Human } \\
\text { influence }\end{array}$ & $\begin{array}{l}\text { Old cultivated, surface } \\
\text { irrigation and drainage } \\
\text { system. }\end{array}$ & $\begin{array}{lr}\text { Relatively } & \text { old }(5,6) \\
\text { or } & \text { recently } \\
\text { cultivated } & (7), \\
\text { surface } & \text { irrigation } \\
\text { and } & \text { drainage } \\
\text { system } & \\
\end{array}$ & $\begin{array}{l}\text { No influence }(8,9,11,12, \\
13,14) \text {, recently cultivated } \\
\text { by sprinkler irrigation (10) }\end{array}$ & $\begin{array}{l}\text { Leveling, surface } \\
\text { irrigation (15) or } \\
\text { sprinkler } \\
\text { irrigation } \\
(16)\end{array}$ & $\begin{array}{l}\text { No influence }(17,20) \text {, } \\
\text { cultivated by sprinkler } \\
\text { irrigation (18) or surface } \\
\text { irrigation (19) }\end{array}$ & No influence \\
\hline $\begin{array}{c}\text { Surface } \\
\text { characteristics }\end{array}$ & $\begin{array}{l}\text { Wide cracks when dry } \\
(1,2,3)\end{array}$ & $\begin{array}{l}\text { Wide cracks when } \\
\text { dry }\end{array}$ & $\begin{array}{l}\text { Few to common gravel } \\
(9,10,11,12,13) \text {, thin sand } \\
\text { sheet }(8) \text {, few gravel and } \\
\text { stones }(14)\end{array}$ & - & $\begin{array}{l}\text { Very few shells (17), } \\
\text { common } \\
\text { vegetation }\end{array}$ & Few gravel and stones \\
\hline $\begin{array}{l}\text { Effective soil } \\
\text { depth and } \\
\text { water table }\end{array}$ & $\begin{array}{l}\text { Very deep; water table } \\
>150 \mathrm{~cm}(1,3,4), 120 \\
\mathrm{~cm}(2) .\end{array}$ & $\begin{array}{l}\text { Moderately deep, } \\
\text { water table } 65 \text { to } 85 \\
\mathrm{~cm} \text { depth }\end{array}$ & $\begin{array}{l}\text { Very deep, } \quad \text { water table } \\
>150 \quad \mathrm{~cm} ; \quad \text { moderately } \\
\text { deep (14). }\end{array}$ & $\begin{array}{l}\text { Very deep, water } \\
\text { table }>150 \mathrm{~cm} .\end{array}$ & $\begin{array}{l}\text { Moderately deep, water } \\
\text { table } 60 \mathrm{~cm}(17) ; 100 \mathrm{~cm} \\
(19) \text {; very deep }(18,20) .\end{array}$ & $\begin{array}{l}\text { Moderately deep }(21) \text {, } \\
\text { shallow }(22) ; \text { water } \\
\text { table }>150 \mathrm{~cm} \text {. }\end{array}$ \\
\hline $\begin{array}{l}\text { Surface soil } \\
\text { layers, } 0-15 \text { to } \\
25 \mathrm{~cm}\end{array}$ & $\begin{array}{l}\text { Dark brown clay texture, } \\
\text { moderate subangular } \\
\text { blocky structure with } \\
\text { hard consistence when } \\
\text { dry }(1,2,3) \text {, sandy clay } \\
\text { loam texture (4) }\end{array}$ & $\begin{array}{l}\text { Very dark grayish } \\
\text { brown clay texture, } \\
\text { moderate to weak } \\
\text { subangular blocky } \\
\text { structure with hard } \\
\text { consistence when } \\
\text { dry. }\end{array}$ & $\begin{array}{l}\text { Yellowish brown to very } \\
\text { pale brown, slightly gravel } \\
\text { loamy sand to sand } \\
\text { texture, massive structure } \\
\text { with soft to loose } \\
\text { consistence when dry. }\end{array}$ & $\begin{array}{l}\text { Light yellowish } \\
\text { brown to } \\
\text { brownish yellow } \\
\text { sand or loamy } \\
\text { sand texture, } \\
\text { single grains and } \\
\text { loose } \\
\text { consistence. }\end{array}$ & $\begin{array}{l}\text { Light yellowish brown } \\
\text { sand with few shells (17); } \\
\text { yellowish brown sandy } \\
\text { loam, massive structure } \\
\text { with soft consistence, few } \\
\text { lime accum. }(18,19,20)\end{array}$ & $\begin{array}{l}\text { Yellowish brown slightly } \\
\text { gravel to gravelly sandy } \\
\text { loam, massive and soft; } \\
\text { few lime accum. (22). }\end{array}$ \\
\hline $\begin{array}{c}\text { Subsurface } \\
\text { layers, } 15 \text { to } 25 \\
\text { cm up to } 100 \\
\text { cm }\end{array}$ & $\begin{array}{l}\text { Dark brown to very dark } \\
\text { grayish brown clay, } \\
\text { strong angular blocky } \\
\text { and clear slickensides up } \\
\text { to } 60-70 \mathrm{~cm} \text {. with sticky } \\
\text { and plastic when wet } \\
(1,2) \text {; brown clay loam } \\
(3) \text { or sandy loam (4), } \\
\text { moderate to weak } \\
\text { subangular blocky with } \\
\text { slightly sticky and plastic } \\
\text { to slightly plastic when } \\
\text { wet. }\end{array}$ & $\begin{array}{l}\text { Very dark gray to } \\
\text { very dark grayish } \\
\text { brown clay with } \\
\text { common to } \\
\text { abundant mottling, } \\
\text { strong angular } \\
\text { blocky and clear } \\
\text { slickensides up to } \\
55 \mathrm{~cm} \text { with sticky to } \\
\text { very stcky } \\
\text { consistence when } \\
\text { wet. }\end{array}$ & $\begin{array}{l}\text { Strong brown to yellowish } \\
\text { red gravelly to very } \\
\text { gravelly loamy sand or } \\
\text { sandy loam }(8,9,10,11 \text {, } \\
14) \text { to sandy clay loam } \\
(12,13) ; \text { massive structure } \\
\text { with soft to hard } \\
\text { consistence when dry; few } \\
\text { to common lime and } \\
\text { gypsum accumulations }\end{array}$ & $\begin{array}{l}\text { Yellow } \\
\text { sand }\end{array}$ & $\begin{array}{l}\text { Light yellowish gray to } \\
\text { brown loose sand with } \\
\text { common to many mottling } \\
\text { (17); yellowish brown to } \\
\text { strong brown sandy clay } \\
\text { loam to loam texture with } \\
\text { common to many } \\
\text { indurated nodules, weak } \\
\text { subangular blocky to } \\
\text { massive structure, many } \\
\text { to common soft lime } \\
\text { accumulation. }\end{array}$ & $\begin{array}{l}\text { Dark reddish gray silty } \\
\text { clay loam, fine and } \\
\text { medium platy structure } \\
\text { up to } 60 \mathrm{~cm} \text { over shale } \\
\text { clay; common gypsum } \\
\text { accum. ( } 21) \text {; strong } \\
\text { brown sandy loam up to } \\
50 \mathrm{~cm} \text { over limestone } \\
(22) \text {. }\end{array}$ \\
\hline $\begin{array}{c}\text { Substratum } \\
>100 \mathrm{~cm} \\
\text { depth }\end{array}$ & $\begin{array}{lr}\text { As above } & (1,2,4) ; \\
\text { yellowish brown sandy } \\
\text { loam and } & \text { weak } \\
\text { subangular } & \text { blocky } \\
\text { structure (3) } & \end{array}$ & $\begin{array}{l}\text { As above with more } \\
\text { stickiness and } \\
\text { plasticity and less } \\
\text { structure grade. }\end{array}$ & $\begin{array}{l}\text { As above with more } \\
\text { coarser texture and less } \\
\text { gravel contents; gravelly } \\
\text { stony layers (14). }\end{array}$ & As above & $\begin{array}{l}\text { As above with less } \\
\text { indurated nodules } \\
\text { contents; many gypsum } \\
\text { accumulation (20). }\end{array}$ & Rock \\
\hline
\end{tabular}


Table 2. Laboratory determinations of the collected soil profile samples.

\begin{tabular}{|c|c|c|c|c|c|c|c|c|c|c|c|c|c|c|}
\hline \multirow{2}{*}{$\begin{array}{l}\text { Prof. No } \\
\text { and } \\
\text { location }\end{array}$} & \multirow{2}{*}{$\begin{array}{l}\text { Depth } \\
\text { (cm) }\end{array}$} & \multirow{2}{*}{$\begin{array}{c}\text { Coarse } \\
\text { Fragments } \\
\%\end{array}$} & \multicolumn{4}{|c|}{ Particle size distribution \% } & \multirow{2}{*}{$\begin{array}{c}{ }^{*} \text { Texture } \\
\text { Class }\end{array}$} & \multirow[b]{2}{*}{ pH } & \multirow[b]{2}{*}{$\begin{array}{c}\text { EC } \\
\mathrm{dS} / \mathrm{m}\end{array}$} & \multirow[b]{2}{*}{$\begin{array}{c}O . M \\
\%\end{array}$} & \multirow{2}{*}{$\begin{array}{c}\text { CEC } \\
\mathrm{cmol} / \mathrm{Kg} \\
\text { soil }\end{array}$} & \multirow[b]{2}{*}{ ESP } & \multirow[b]{2}{*}{$\begin{array}{c}\mathrm{CaCO} 3 \\
\%\end{array}$} & \multirow[b]{2}{*}{$\begin{array}{c}\text { Gypsum } \\
\%\end{array}$} \\
\hline & & & $\begin{array}{c}\text { Coarse } \\
\text { sand }\end{array}$ & $\begin{array}{l}\text { Fine } \\
\text { sand }\end{array}$ & Silt & clay & & & & & & & & \\
\hline \multicolumn{15}{|c|}{ Recent Nile alluvial soils (Nile Delta) } \\
\hline \multirow{3}{*}{$\begin{array}{c}1 \\
\text { El-Monofia Gov. }\end{array}$} & $0-20$ & - & 2.7 & 11.4 & 32.4 & 53.5 & C & 7.9 & 1.2 & 1.6 & 51 & 4.5 & 2.4 & 1.1 \\
\hline & $20-60$ & - & 1.9 & 11.8 & 30.1 & 56.2 & C & 7.8 & 1.1 & 1.1 & 60 & 3.7 & 1.4 & 0.9 \\
\hline & $60-150$ & - & 1.8 & 7.3 & 33.5 & 57.4 & C & 7.7 & 1.5 & 1.0 & 55 & 5.4 & 1.7 & 1.4 \\
\hline \multirow{3}{*}{$\begin{array}{l}2 \\
\text { Kafr Elshikh Gov }\end{array}$} & $0-20$ & - & 1.5 & 11.9 & 31.6 & 55.0 & $\mathrm{C}$ & 7.9 & 1.3 & 1.4 & 55 & 6.6 & 2.5 & 1.2 \\
\hline & $20-70$ & - & 0.85 & 6.55 & 29.1 & 63.5 & C & 7.8 & 2.09 & 0.86 & 81 & 7.1 & 2.5 & 0.7 \\
\hline & $70-120$ & - & 0.66 & 3.84 & 28.4 & 67.1 & $\mathrm{C}$ & 7.6 & 3.26 & 0.88 & 68 & 8.9 & 1.9 & 0.8 \\
\hline \multirow{4}{*}{$\begin{array}{c}3 \\
\text { El-Qalubia } \\
\text { Gov. }\end{array}$} & $0-20$ & - & 6.5 & 23.6 & 25.7 & 44.2 & $\mathrm{C}$ & 7.8 & 2.1 & 1.8 & 47 & 3.5 & 1.5 & 1.0 \\
\hline & $20-50$ & - & 7.1 & 24.2 & 33.4 & 35.3 & $\mathrm{CL}$ & 7.7 & 1.6 & 1.1 & 40 & 3.5 & 1.6 & 0.8 \\
\hline & $50-100$ & - & 12.4 & 26.7 & 30.1 & 30.8 & $\mathrm{CL}$ & 7.8 & 1.5 & 1.1 & 36 & 1.9 & 2.0 & 0.8 \\
\hline & $100-150$ & - & 22.6 & 39.6 & 20.3 & 17.5 & SL & 7.5 & 1.4 & 0.9 & 29 & 1.8 & 1.9 & 0.7 \\
\hline \multirow{3}{*}{$\begin{array}{c}4 \\
\text { El-Monofia Gov. }\end{array}$} & $0-25$ & - & 27.3 & 26.3 & 20.8 & 25.6 & $\mathrm{SCL}$ & 7.9 & 0.9 & 1.6 & 32 & 2.4 & 1.8 & 0.9 \\
\hline & $25-55$ & - & 45.1 & 19.5 & 15.5 & 17.4 & $\mathrm{SL}$ & 7.8 & 0.8 & 0.8 & 27 & 2.6 & 1.5 & 1.6 \\
\hline & $55-150$ & - & 47.6 & 20.8 & 21.4 & 10.2 & $\mathrm{SL}$ & 8.0 & 0.6 & 0.7 & 21 & 1.6 & 1.2 & 0.6 \\
\hline \multicolumn{15}{|c|}{ Fluvio-Marine soils } \\
\hline \multirow{3}{*}{$\begin{array}{c}5 \\
\text { Idkou }\end{array}$} & $0-20$ & - & 1.1 & 23.0 & 25.6 & 50.3 & $\mathrm{C}$ & 7.8 & 3.7 & 2.1 & 44 & 14.6 & 4.8 & 2.2 \\
\hline & $20-50$ & 4 & 2.2 & 14.5 & 36.8 & 46.5 & $\mathrm{C}$ & 8.2 & 6.3 & 1.1 & 51 & 16.2 & 3.4 & 2.9 \\
\hline & $50-85$ & - & 0.8 & 3.8 & 45.3 & 50.1 & $\mathrm{SiC}$ & 8.1 & 5.7 & 1.1 & 52 & 18.1 & 3.2 & 1.1 \\
\hline \multirow{3}{*}{$\begin{array}{c}6 \\
\text { Kafr Elshikh Gov }\end{array}$} & $0-20$ & - & 2.5 & 19.7 & 33.2 & 44.6 & $\mathrm{C}$ & 8.0 & 4.7 & 1.7 & 51 & 11.2 & 0.6 & 0.9 \\
\hline & $20-55$ & - & 0.6 & 20.5 & 31.5 & 47.4 & C & 7.9 & 5.2 & 1.1 & 49 & 13.1 & 1.7 & 1.1 \\
\hline & $55-80$ & - & 0.8 & 16.2 & 29.8 & 53.2 & C & 7.9 & 6.4 & 0.9 & 52 & 14.6 & 1.1 & 0.8 \\
\hline 7 & $0-20$ & - & 1.7 & 11.6 & 29.3 & 57.4 & $\mathrm{C}$ & 8.4 & 15.4 & 2.3 & 49 & 18.1 & 7.6 & 0.2 \\
\hline El-Hasaniya & $20-45$ & - & 0.8 & 4.8 & 29.2 & 65.2 & $\mathrm{C}$ & 8.2 & 5.5 & 1.4 & 55 & 18.0 & 4.0 & 0.4 \\
\hline Plain & $45-65$ & - & 0.9 & 5.6 & 27.4 & 66.1 & $\mathrm{C}$ & 8.3 & 7.2 & 0.9 & 54 & 22.3 & 1.2 & 0.1 \\
\hline \multicolumn{15}{|c|}{ Old alluvial plain soils } \\
\hline 8 & $0-15$ & 5 & 56.3 & 24.4 & 12.8 & 6.5 & LS & 7.9 & 1.5 & 0.3 & 10 & 1.5 & 2.2 & 0.3 \\
\hline Ismaeilya & $15-60$ & 20 & 60.4 & 24.0 & 6.5 & 9.1 & LS & 8.1 & 5.5 & 0.4 & 12 & 4.1 & 5.3 & 0.5 \\
\hline Gov. & $60-150$ & 8 & 70.2 & 12.8 & 9.5 & 7.5 & LS & 8.3 & 6.1 & 0.4 & 9 & 3.5 & 3.2 & 1.2 \\
\hline \multirow{4}{*}{$\begin{array}{c}9 \\
\text { Ismaeilya } \\
\text { Gov. }\end{array}$} & $0-15$ & 10 & 31.3 & 54.2 & 8.2 & 6.3 & LS & 7.8 & 13.5 & 0.4 & 8 & 3.8 & 7.4 & 0.15 \\
\hline & $15-60$ & 45 & 40.2 & 50.6 & 5.1 & 4.1 & $\mathrm{~S}$ & 8.1 & 11.4 & 0.2 & 5 & 2.5 & 26.1 & 0.22 \\
\hline & $60-100$ & 30 & 36.6 & 45.8 & 9.2 & 8.4 & LS & 7.9 & 12.8 & 0.3 & 9 & 2.6 & 19.2 & 2.3 \\
\hline & $100-150$ & 20 & 37.9 & 50.7 & 5.2 & 6.2 & $\mathrm{~S}$ & 8.0 & 4.4 & 0.1 & 4 & 1.9 & 15.4 & 2.8 \\
\hline \multirow{4}{*}{$\begin{array}{c}10 \\
\text { El-Salhia } \\
\text { project }\end{array}$} & $0-15$ & 3 & 40.2 & 41.9 & 7.8 & 10.1 & LS & 7.9 & 2.9 & 1.1 & 14 & 6.6 & 6.5 & - \\
\hline & $15-50$ & 10 & 45.7 & 37.4 & 10.4 & 6.5 & LS & 8.0 & 3.1 & 0.6 & 9 & 4.2 & 7.1 & 0.6 \\
\hline & $50-90$ & 35 & 33.6 & 38.6 & 17.5 & 10.3 & SL & 8.1 & 4.2 & 0.4 & 12 & 5.1 & 17.2 & 0.9 \\
\hline & $90-150$ & 10 & 42.3 & 39.5 & 8.4 & 9.8 & LS & 7.8 & 2.5 & 0.5 & 8 & 3.8 & 9.3 & 0.4 \\
\hline
\end{tabular}

*Texturel class: (C) clay, (CL) clay loam, (SiC) silty clay, (SCL) sandy clay loam, (SL) sandy loam, (LS) loamy sand, (S) san 
Table 2. Cont.

\begin{tabular}{|c|c|c|c|c|c|c|c|c|c|c|c|c|c|c|}
\hline \multirow{2}{*}{$\begin{array}{l}\text { Prof. No. } \\
\text { and } \\
\text { landform }\end{array}$} & \multirow{2}{*}{$\begin{array}{l}\text { Depth } \\
\text { (cm) }\end{array}$} & \multirow{2}{*}{$\begin{array}{c}\text { Coarse } \\
\text { Fragments } \\
\%\end{array}$} & \multicolumn{4}{|c|}{ Particle size distribution \% } & \multirow{2}{*}{$\begin{array}{l}* \text { Texture } \\
\text { Class }\end{array}$} & \multirow[b]{2}{*}{ pH } & \multirow[b]{2}{*}{$\begin{array}{c}\mathrm{EC} \\
\mathrm{dS} / \mathrm{m}\end{array}$} & \multirow[b]{2}{*}{$\begin{array}{l}\text { O.M } \\
\%\end{array}$} & \multirow{2}{*}{$\begin{array}{c}\mathrm{CEC} \\
\mathrm{cmol} / \mathrm{Kg} \\
\text { soil }\end{array}$} & \multirow[b]{2}{*}{ ESP } & \multirow[b]{2}{*}{$\begin{array}{c}\mathrm{CaCO} 3 \\
\%\end{array}$} & \multirow[b]{2}{*}{$\begin{array}{c}\text { Gypsum } \\
\%\end{array}$} \\
\hline & & & $\begin{array}{c}\text { Coarse } \\
\text { sand }\end{array}$ & $\begin{array}{l}\text { Fine } \\
\text { sand }\end{array}$ & Silt & clay & & & & & & & & \\
\hline \multicolumn{15}{|c|}{ Old alluvial plain soils } \\
\hline & $0-20$ & 5 & 34.2 & 49.4 & 7.1 & 9.3 & LS & 7.7 & 32.3 & 0.7 & 8 & 11.4 & 3.0 & 0.1 \\
\hline 11 & $20-40$ & 15 & 62.6 & 22.0 & 6.3 & 9.1 & LS & 7.6 & 30.7 & 0.9 & 6 & 9.6 & 3.5 & 0.4 \\
\hline $10^{\text {th }}$ of Ramdan & $40-80$ & 40 & 42.3 & 36.7 & 5.8 & 15.2 & $\mathrm{SL}$ & 7.5 & 39.4 & 0.4 & 11 & 12.1 & 2.4 & 0.7 \\
\hline & $80-150$ & 15 & 46.6 & 42.5 & 7.5 & 3.4 & $\mathrm{~S}$ & 7.5 & 16.1 & 0.2 & 5 & 8.2 & 1.2 & 2.3 \\
\hline & $0-15$ & 10 & 31.4 & 49.9 & 10.2 & 8.5 & LS & 7.7 & 11.4 & 0.8 & 8 & 8.5 & 7.3 & 1.1 \\
\hline El-Sadat & $15-45$ & 20 & 33.2 & 35.1 & 18.1 & 13.6 & SL & 7.9 & 20.5 & 0.5 & 6 & 10.4 & 16.6 & 2.3 \\
\hline City area & $45-95$ & 42 & 27.8 & 31.5 & 15.4 & 25.3 & $\mathrm{SCL}$ & 7.8 & 28.6 & 0.4 & 16 & 12.5 & 15.4 & 9.7 \\
\hline & $95-150$ & 10 & 42.4 & 39.7 & 12.1 & 5.8 & LS & 8.0 & 21.2 & 0.2 & 6 & 10.6 & 7.6 & 1.2 \\
\hline 13 & $0-25$ & 30 & 18.1 & 51.3 & 14.2 & 16.4 & $\mathrm{SI}$ & 7.8 & 10.3 & 0.4 & 12 & 5.7 & 6.1 & 0.9 \\
\hline Wadi & $25-80$ & 20 & 12.3 & 35.7 & 21.8 & 30.2 & $\mathrm{SCL}$ & 7.6 & 8.6 & 0.2 & 18 & 7.4 & 3.3 & 0.4 \\
\hline El-Farigh & $80-150$ & 10 & 20.5 & 50.3 & 11.4 & 17.8 & $\mathrm{SL}$ & 7.7 & 11.2 & 0.1 & 10 & 8.1 & 2.4 & 1.1 \\
\hline 14 & $0-20$ & 10 & 48.3 & 34.4 & 8.2 & 9.1 & LS & 7.5 & 17.7 & 0.1 & 9 & 9.2 & 23.4 & 1.5 \\
\hline Wadi El-Farigh & $20-70$ & 40 & 45.6 & 29.3 & 9.8 & 15.3 & SL & 7.9 & 11.3 & 0.09 & 13 & 8.3 & 16.2 & 1.7 \\
\hline \multicolumn{15}{|c|}{ Windblown sand soils } \\
\hline 15 & $0-20$ & - & 56.7 & 32.8 & 3.1 & 7.4 & $\mathrm{~S}$ & 7.9 & 1.3 & 1.1 & 8 & 6.7 & 4.1 & 0.2 \\
\hline El-Bostan & $20-60$ & - & 59.6 & 31.1 & 3.8 & 5.5 & s & 7.8 & 1.0 & 0.3 & 4 & 5.1 & 3.5 & 0.1 \\
\hline area & $60-150$ & - & 59.8 & 31.2 & 3.6 & 5.4 & $\mathrm{~s}$ & 7.8 & 1.2 & 0.4 & 4 & 5.2 & 4.4 & 0.1 \\
\hline 16 & $0-15$ & - & 66.7 & 18.8 & 5.4 & 9.1 & LS & 8.03 & 3.7 & 1.05 & 19 & 10.1 & 1.02 & 0.1 \\
\hline El-Salhia area & $15-150$ & - & 75.1 & 16.7 & 3.3 & 4.9 & $\mathrm{~S}$ & 8.12 & 1.9 & 0.54 & 5 & 7.4 & 1.55 & - \\
\hline \multicolumn{15}{|c|}{ Coastal plain soils } \\
\hline & $0-15$ & 4 & 30.4 & 65.1 & 2.3 & 2.2 & $\mathrm{~S}$ & 7.0 & 5.6 & 0.4 & 3 & 19.3 & 0.2 & 0.6 \\
\hline Kafr Flahikh Gov & $15-35$ & - & 33.6 & 59.9 & 3.1 & 3.4 & $\mathrm{~s}$ & 7.5 & 35.5 & 0.3 & 4 & 21.1 & 0.2 & 0.5 \\
\hline Kafr Elshikh Gov & $35-60$ & - & 35.2 & 54.9 & 3.8 & 6.1 & $\mathrm{~s}$ & 7.5 & 40.8 & 0.2 & 4 & 17.6 & 0.5 & 0.4 \\
\hline 18 & $0-30$ & 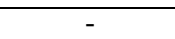 & 40.1 & 32.1 & 14.5 & 13.3 & $\mathrm{SL}$ & 7.7 & 1.8 & 0.55 & 13 & 2.6 & 10.4 & 3.3 \\
\hline Nubariya & $30-70$ & 10 & 32.4 & 28.8 & 16.2 & 22.6 & $\mathrm{SCL}$ & 8.0 & 1.9 & 0.40 & 18 & 5.8 & 16.6 & 2.1 \\
\hline Res. Station & $70-150$ & 4 & 28.3 & 25.2 & 20.4 & 26.1 & $\mathrm{SCL}$ & 8.1 & 2.7 & 0.40 & 19 & 5.3 & 20.8 & 2.5 \\
\hline 19 & $0-25$ & - & 49.6 & 27.1 & 12.8 & 10.5 & SL & 7.9 & 3.6 & 0.65 & 8 & 8.4 & 18.6 & 1.9 \\
\hline Nubariya & $25-60$ & 5 & 30.3 & 51.1 & 10.2 & 8.4 & LS & 7.9 & 2.5 & 0.32 & 5 & 11.5 & 20.2 & 3.6 \\
\hline area & $60-100$ & 20 & 35.4 & 26.2 & 15.2 & 23.2 & $\mathrm{SCL}$ & 8.0 & 4.4 & 0.24 & 16 & 10.1 & 30.3 & 4.8 \\
\hline & $0-15$ & 4 & 25.4 & 47.9 & 14.4 & 12.3 & $\mathrm{SL}$ & 7.6 & 7.2 & 0.3 & 9 & 6.6 & 31.5 & 0.3 \\
\hline 20 & $15-45$ & 20 & 29.6 & 33.7 & 15.1 & 21.6 & $\mathrm{SCL}$ & 7.6 & 8.8 & 0.3 & 14 & 7.1 & 40.2 & 0.2 \\
\hline South El-Nasr canal & $45-80$ & 40 & 19.2 & 26.1 & 32.5 & 22.2 & $\mathrm{~L}$ & 7.6 & 11.5 & 0.25 & 13 & 7.4 & 30.1 & 1.9 \\
\hline & $80-150$ & 3 & 16.2 & 23.1 & 35.3 & 25.4 & L & 7.7 & 13.6 & 0.05 & 14 & 7.3 & 26.3 & 15.5 \\
\hline \multicolumn{15}{|c|}{ Miscellaneous land types } \\
\hline 21 & $0-15$ & 5 & 44.2 & 26.1 & 16.1 & 13.6 & $\mathrm{SL}$ & 7.7 & 2.6 & 0.2 & 11 & 7.1 & 6.3 & 1.1 \\
\hline $6^{\text {th }}$ of October & $15-60$ & 20 & 1.6 & 13.3 & 48.2 & 36.9 & $\mathrm{SiCL}$ & 8.3 & 18.4 & 0.3 & 40 & 14.2 & 0.9 & 10.5 \\
\hline 22 & $0-20$ & 20 & 40.6 & 32.5 & 12.6 & 14.3 & SL & 7.1 & 35.4 & 0.3 & 13 & 17.4 & 3.5 & 2.3 \\
\hline $10^{\text {th }}$ of Ramadan & $20-50$ & 50 & 48.4 & 27.7 & 11.2 & 12.7 & SL & 7.2 & 40.5 & 0.3 & 11 & 19.8 & 8.8 & 2.1 \\
\hline
\end{tabular}

*Texturel class: (C) clay, (CL) clay loam, (SiC) silty clay, (SCL) sandy clay loam, (SL) sandy loam, (LS) loamy sand, (S) sand 
Table 3. Soil Taxonomic units of the investigated soil profiles(according to USDA,2014)

\begin{tabular}{|c|c|}
\hline $\begin{array}{l}\text { Profile } \\
\text { No. }\end{array}$ & Taxonomic unit \\
\hline \multicolumn{2}{|r|}{ Recent Nile alluvial soils (Nile Delta) } \\
\hline $\begin{array}{l}1 \\
2 \\
3 \\
4\end{array}$ & $\begin{array}{l}\text { Typic Haplotorrerts; fine, smectitic, thermic. } \\
\text { Typic Haplotorrerts; very-fine, smectitic, thermic. } \\
\text { Typic Torrifluvents; fine-loamy, mixed, superactive, calcareous, thermic. } \\
\text { Typic Torrifluvents; coarse-loamy, mixed, superactive, calcareous, thermic. }\end{array}$ \\
\hline \multicolumn{2}{|r|}{ Fluvio-Marine soils } \\
\hline $\begin{array}{l}5 \\
6 \\
7\end{array}$ & $\begin{array}{l}\text { Sodic Endoaquerts; fine, smectitic, thermic. } \\
\text { Typic Endoaquerts; fine, smectitic, thermic. } \\
\text { Sodic Endoaquerts; very-fine, smectitic, thermic. }\end{array}$ \\
\hline \multicolumn{2}{|r|}{ Old alluvial soils } \\
\hline $\begin{array}{l}8 \\
9 \\
10 \\
11 \\
12 \\
13 \\
14\end{array}$ & $\begin{array}{l}\text { Typic Torriorthents; sandy, mixed, hyperthermic. } \\
\text { Typic Haplocalcids; sandy-skeletal, mixed, hyperthermic . } \\
\text { Typic Haplocalcids; loamy-skeletal, mixed, superactive, thermic. } \\
\text { Typic Torriorthents; loamy-skeletal, mixed, superactive, calcareous, thermic. } \\
\text { Typic Calcigypsids; loamy-skeletal, mixed, superactive, thermic . } \\
\text { Typic Torriorthents; fine-loamy, mixed, superactive, calcareous, thermic. } \\
\text { Typic Haplocalcids; loamy-skeletal over fragmental, mixed, superactive, thermic. }\end{array}$ \\
\hline \multicolumn{2}{|r|}{ Windblown sand soils } \\
\hline $\begin{array}{l}15 \\
16\end{array}$ & $\begin{array}{l}\text { Typic Torripsamments; siliceous, thermic. } \\
\text { Typic Torripsamments; siliceous, thermic. }\end{array}$ \\
\hline \multicolumn{2}{|r|}{ Coastal plain soils } \\
\hline $\begin{array}{l}17 \\
18 \\
19 \\
20\end{array}$ & $\begin{array}{l}\text { Sodic Psammaquents; siliceous, thermic. } \\
\text { Typic Haplocalcids; fine-loamy, mixed, superactive, thermic. } \\
\text { Typic Haplocalcids; sandy over loamy, mixed, superactive, thermic. } \\
\text { Typic Calcigypsids; fine-loamy, carbonatic, thermic. }\end{array}$ \\
\hline \multicolumn{2}{|r|}{ Miscellaneous land types } \\
\hline $\begin{array}{l}21 \\
22\end{array}$ & $\begin{array}{l}\text { Typic Haplogypsids; fine-loamy, mixed, superactive, hyperthermic. } \\
\text { Lithic Torriorthents; loamy-skeletal, superactive, calcareous, thermic. }\end{array}$ \\
\hline
\end{tabular}


Table 4. Suitability classes of soil profiles by using the investigated methods.

\begin{tabular}{|c|c|c|c|c|c|c|c|c|c|c|c|c|c|c|}
\hline \multirow{4}{*}{$\begin{array}{c}\text { Profile } \\
\text { No. }\end{array}$} & \multicolumn{4}{|c|}{ Descriptive methods } & \multicolumn{10}{|c|}{ Parametric methods } \\
\hline & \multicolumn{2}{|c|}{ FAO } & \multicolumn{2}{|c|}{ USBR } & \multicolumn{4}{|c|}{ Storie } & \multicolumn{4}{|c|}{ Sys and Verheye } & \multirow{2}{*}{\multicolumn{2}{|c|}{$\begin{array}{c}\text { Sys et al. } \\
\text { (1991) }\end{array}$}} \\
\hline & \multirow{2}{*}{$\begin{array}{c}(1979 \\
)\end{array}$} & \multirow{2}{*}{$\begin{array}{c}\text { Sys et } \\
\text { al. } \\
\text { (1991) }\end{array}$} & \multirow{2}{*}{$\begin{array}{c}\text { Griffiths } \\
\text { (1975) }\end{array}$} & \multirow{2}{*}{$\begin{array}{c}\text { FAO } \\
(1979)\end{array}$} & \multicolumn{2}{|c|}{ Nelson (1963) } & \multicolumn{2}{|c|}{ (1978) } & \multicolumn{2}{|c|}{ (1974) } & \multicolumn{2}{|c|}{ (1978) } & & \\
\hline & & & & & $\mathbf{C i}$ & Class & $\mathbf{C i}$ & Class & $\mathbf{C i}$ & Class & $\mathbf{C i}$ & Class & $\mathbf{C i}$ & Class \\
\hline \multicolumn{15}{|c|}{ Recent Nile alluvial soils (Nile Delta) } \\
\hline 1 & S2 & S2 & Class 2 & Class 2 & 71.9 & B & 68.4 & 2 & 76.0 & II & 70.7 & $\mathrm{~S} 2$ & 62.9 & $\mathrm{~S} 2$ \\
\hline 2 & S3 & S3 & Class 3 & Class 3 & 64.3 & $\mathrm{C}$ & 57.8 & 3 & 59.9 & III & 52.6 & S3 & 44.5 & S3 \\
\hline 3 & S1 & S1 & Class 1 & Class 1 & 81.9 & $\mathrm{~B}$ & 80.0 & 1 & 95.0 & I & 86.3 & S1 & 84.2 & S1 \\
\hline 4 & S1 & S2 & Class 1 & Class 1 & 84.6 & $\mathrm{~A}$ & 100.0 & 1 & 71.3 & II & 75.0 & S1 & 60.0 & $\mathrm{~S} 2$ \\
\hline \multicolumn{15}{|c|}{ Fluvio - Marine soils } \\
\hline 5 & S3 & S3 & Class 3 & Class 3 & 42.0 & $\mathrm{D}$ & 47.6 & 3 & 39.4 & IV & 48.5 & S3 & 37.9 & S3 \\
\hline 6 & S2 & S3 & Class 3 & Class 2 & 42.0 & D & 51.0 & 3 & 45.2 & III & 46.0 & S3 & 36.3 & S3 \\
\hline 7 & S3 & N1 & Class 3 & Class 3 & 37.3 & $\mathrm{D}$ & 44.8 & 3 & 27.3 & $\mathrm{~V}$ & 32.1 & S3 & 22.9 & N1 \\
\hline \multicolumn{15}{|c|}{ Old alluvial plain soils } \\
\hline 8 & S3 & S3 & Class 3 & Class 3 & 59.4 & $\mathrm{C}$ & 72.2 & 2 & 47.2 & III & 42.4 & S3 & 34.8 & S3 \\
\hline 9 & S3 & S3 & Class 3 & Class 3 & 40.9 & $\mathrm{D}$ & 34.0 & 4 & 28.8 & $\mathrm{~V}$ & 25.0 & S3 & 11.6 & N1 \\
\hline 10 & S3 & S3 & Class 3 & Class 3 & 69.6 & $B$ & 57.6 & 3 & 51.3 & III & 50.8 & $\mathrm{~S} 2$ & 29.3 & S3 \\
\hline 11 & N1 & S3 & Class 3 & Class 5 & 54.7 & $\mathrm{C}$ & 57.8 & 3 & 36.8 & IV & 32.6 & $\mathrm{~S} 3$ & 24.3 & $\mathrm{~N} 1$ \\
\hline 12 & N1 & S2 & Class 2 & Class 5 & 54.7 & $\mathrm{C}$ & 54.7 & 3 & 51.8 & III & 50.0 & $\mathrm{~S} 2$ & 24.2 & N1 \\
\hline 13 & S3 & S2 & Class 2 & Class 3 & 58.0 & $\mathrm{C}$ & 56.5 & 3 & 61.4 & II & 57.2 & $\mathrm{~S} 2$ & 38.7 & S3 \\
\hline 14 & S3 & S3 & Class 2 & Class 3 & 44.6 & $\mathrm{D}$ & 54.0 & 3 & 34.4 & IV & 28.3 & S3 & 19.1 & N1 \\
\hline \multicolumn{15}{|c|}{ Windblown sand soils } \\
\hline 15 & Sc & N1 & Class 4 & Class 4 & 41.7 & D & 60.0 & 2 & 25.7 & $\mathrm{~V}$ & 25.7 & S3 & 27.0 & S3 \\
\hline 16 & Sc & N1 & Class 4 & Class 4 & 50.6 & $\mathrm{D}$ & 57.0 & 3 & 27.6 & V & 26.1 & S3 & 25.9 & S3 \\
\hline \multicolumn{15}{|c|}{ Coastal plain soils } \\
\hline 17 & N1 & N1 & Class 5 & Class 5 & 33.6 & D & 28.5 & 4 & 8.0 & $\mathrm{~V}$ & 8.0 & N2 & 8.5 & N1 \\
\hline 18 & S2 & S2 & Class 2 & Class 2 & 67.0 & $\mathrm{C}$ & 68.9 & 2 & 83.6 & I & 85.8 & S1 & 58.1 & $\mathrm{~S} 2$ \\
\hline 19 & S2 & S2 & Class 2 & Class 2 & 67.0 & $\mathrm{C}$ & 60.8 & 2 & 58.1 & III & 53.6 & S2 & 42.9 & S3 \\
\hline 20 & S3 & S2 & Class 2 & Class 3 & 58.1 & $\mathrm{C}$ & 62.0 & 2 & 57.5 & III & 55.4 & S2 & 34.3 & S3 \\
\hline \multicolumn{15}{|c|}{ Miscellaneous Land types } \\
\hline 21 & Sc & S3 & Class 4 & Class 4 & 29.7 & $\mathrm{E}$ & 27.4 & 4 & 30.0 & IV & 32.2 & S3 & 31.6 & S3 \\
\hline 22 & N1 & S3 & Class 4 & Class 5 & 30.6 & $\mathrm{D}$ & 27.0 & 4 & 18.0 & $\mathrm{~V}$ & 16.0 & N1 & 8.9 & N1 \\
\hline
\end{tabular}




\section{Where :}

\section{FAO (1979)}

S1 Highly suitable

Moderately suitable

Marginally Suitable

Conditionally Suitable

Currently not Suitable

Potentially not suitable

\section{Storie (Nelson, 1963)}

\begin{tabular}{|c|c|}
\hline Class & Capability index (Ci) \\
\hline$A$ & $85-100$ \\
\hline B & $70-84$ \\
\hline C & $55-69$ \\
\hline D & $30-54$ \\
\hline $\mathrm{E}$ & $<30$ \\
\hline
\end{tabular}

\section{USBR related to FAO (1979)}

Class 1 Highly suitable

Class 2 Moderately suitable

Class 3 Marginally suitable

Class 4 Special use land

Class 5 Non- arable (requires further studies)

\section{Storie (1978)}

$\begin{array}{lc}\text { Class } & (\mathrm{Ci}) \\ 1 \text { (Excellent) } & 80-100 \\ 2 \text { (Good) } & 60-79 \\ 3 \text { (Fair) } & 40-59 \\ 4 \text { (Poor) } & 20-39 \\ 5 \text { (Very Poor) } & 10-19 \\ 6 \text { (Non agricultural) } & <10\end{array}$

\section{USBR (Griffiths, 1975)}

Class 1 Highly suitable Class 2 Suitable Class 3 Moderately suitable

Class 4 Marginally suitable Class 5 Unsuitable

\section{Sys and Verheye (1974)}

\begin{tabular}{cc}
\multicolumn{1}{c}{ Class } & $(\mathrm{Ci})$ \\
I (Excellent) & $>80$ \\
II (Suitable) & $60-80$ \\
III (Slightly suit.) & $45-60$ \\
IV (Almost unsuitable) & $30-45$ \\
$\quad$ V (Unsuitable) & $<30$
\end{tabular}

Sys and Verheye (1978) \& Sys et al. (1991)

\section{$\underline{\text { Class }}$}

S1 (Highly suitable)

S2 (Moderately suitable)

S3 (Marginally suitable)

N1 (Currently not suitable)

N2 (Potentially not suitable)

$$
\begin{gathered}
\quad \frac{(\mathrm{Ci})}{>75} \\
50-75 \\
25-50 \\
<25
\end{gathered}
$$




\section{b- Parametric methods:}

- The potential suitability of the two Storie methods indicate different grade in $50 \%$ of the sites. The differences are mainly due to the relatively high rating of the character of soil profile in the Stroie method (Storie, 1978) and also for the range of the capability index grades.

- The current suitability of the Sys methods indicate similar classes in only 6 out of the 22 examined sites most of them in the Nile Delta soils. The proposed rating of Sys et. al. (1991) records the lowest suitability indices while Sys and Verheye (1974) indicates the highest one.

\section{c- Descriptive and parametric methods:}

- 14 sites indicate almost similar classes between Griffiths (1975) and Storie (1978). The differences were observed in 8 sites represent windblown sand and most of the old alluvial soils. The different are more clear with Storie (1963) method.

- 11 sites of Sys and Verheye (1974), 14 sites of both Sys and Verheye (1978) and Sys et. al. (1991) methods indicate almost similar classes of the descriptive FAO (1979), but with no clear trend observed for soil nature or profile numbers.

- The descriptive method regarding to land qualities and that of parametric individual soil characteristics (Sys et. al, 1991) indicate different classes in 10 sites and the more suitability classes were observed in the descriptive one.

According to the differences of the above-mentioned results, it could be concluded that the rating values in some soil factors need more modification to reduce the gap between them.

\section{Proposed modifications of soil rating:}

- The proposed rating of the parametric land suitability evaluation for irrigated agriculture is listed in Table (5). It depends mainly on the principles of land classification in arid and semi-arid regions that discussed by the working group acting at the International Training Center for Post-graduate Soil Scientists (Sys and Verheye, 1972). Also, the concepts of FAO Framework (1976) and Storie methods (1954 and 1978) as well as Sys et. al. (1991 and 1993) were taken into consideration. The proposed modifications of some soil factors imply the respect of the following:

- The rating values are assigned to the characteristics of the soil itself; including the more stable characteristics (factor a), the relatively less stable characteristics (factor $b$ ), surface characteristics (factor $c$ ) and the miscellaneous (factor $x$ ). The later factor which includes erosion hazard and soil fertility could be used only if necessary. The fertility criteria are not directly considered indeed, as weathering stage of arid lands is always in a recent stage, the apparent cation exchange capacity is high to medium. Base saturation is always high and a disturbed cation balance is going to be considered by other characteristics (Sys, 1980). 
Table 5. The proposed land suitability rating chart for irrigated agriculture.

\begin{tabular}{|c|c|c|c|c|}
\hline \multicolumn{5}{|c|}{ Factor (a) : More stable soil profile characteristics } \\
\hline \multirow[t]{2}{*}{ Soil characteristics } & \multicolumn{4}{|c|}{ Rating scale \% } \\
\hline & $\begin{array}{l}\text { Surface } \\
\text { irrigation }\end{array}$ & $\begin{array}{l}\text { Sprinkler } \\
\text { Or drip } \\
\text { irrigation }\end{array}$ & & Specific crops \\
\hline $\begin{array}{l}\text {-Soil texture } \\
\text { including gravel: } \\
\text { Fine-loamy } \\
\text { CL,SiCL (18-35\% clay)- } \\
\text { SCL-L, SiL, SL (>18\% } \\
\text { clay). } \\
\text { With <15\% coarse } \\
\text { fragm. } \\
\quad 15-35 \% \\
\end{array}$ & $\begin{array}{l}100-90 \\
90-80\end{array}$ & $100-90$ & $\begin{array}{l}100- \\
90\end{array}$ & $\begin{array}{l}\text { Groundnuts,sesame,carrot,onion, } \\
\text { green pepper, cabbage, watermelon, }\end{array}$ \\
\hline $35-60 \%$ & $80-70$ & $90-80$ & & potato, olives, citrus, mango. \\
\hline $60-90 \%$ & $70-50$ & $80-60$ & $90-70$ & \\
\hline $\begin{array}{l}\text { Fine - clayey } \\
\mathrm{CL}, \mathrm{SiCL}(>35 \% \text { clay)-C } \\
(<60 \%)-\mathrm{SiC}, \mathrm{SC} \\
\text { With }<15 \% \text { coarse } \\
\text { fragm. } \\
\qquad 15-35 \%\end{array}$ & \multicolumn{2}{|c|}{$\begin{array}{l}90-80 \\
80-75\end{array}$} & $\begin{array}{l}100- \\
90 \\
\end{array}$ & \multirow{2}{*}{$\begin{array}{l}\text { Rice, sugar beet, sugar cane, clover, } \\
\text { alfalafa, cotton, soya, barley, wheat, } \\
\text { maize, sorghum, cowpea, beans, pea, } \\
\text { sunflower, guava, banana. }\end{array}$} \\
\hline $\begin{array}{l}35-60 \% \\
60-90 \%\end{array}$ & & & $\begin{array}{l}90-80 \\
80-60\end{array}$ & \\
\hline $\begin{array}{l}\text { Coarse -loamy } \\
\mathrm{L} \text {, SiL,SL( }<18 \% \text { clay) } \\
\text { With }<15 \% \text { coarse } \\
\text { fragm. } \\
\qquad \begin{array}{l}15-35 \% \\
35-60 \% \\
60-90 \%\end{array}\end{array}$ & $\begin{array}{l}85-70 \\
70-60 \\
60-50 \\
50-30\end{array}$ & $\begin{array}{l}90-80 \\
80-70 \\
70-60 \\
60-40\end{array}$ & $\begin{array}{l}95-85 \\
85-75 \\
75-65 \\
65-50\end{array}$ & $\begin{array}{l}\text { Groundnuts, sesame, carrot, onion, } \\
\text { green pepper, cabbage, watermelon, } \\
\text { potato, olives, mango, citrus. }\end{array}$ \\
\hline $\begin{array}{l}\text { Very fine-clayey } \\
\mathrm{C}(>60 \%) \\
\text { With }<15 \% \text { coarse } \\
\text { fragm. } \\
\qquad \begin{array}{c}15-35 \% \\
35-60 \% \\
60-90 \%\end{array}\end{array}$ & \multicolumn{2}{|c|}{$\begin{array}{l}80-70 \\
70-65 \\
65-60 \\
60-50\end{array}$} & $\begin{array}{l}100-95 \\
95-90 \\
90-85 \\
85-70\end{array}$ & For rice \\
\hline $\begin{array}{l}\text { Sandy } \\
\text { LfS, LS, LCS, fS, S, cS. } \\
\text { With }<15 \% \text { coarse } \\
\text { fragm. } \\
\qquad \begin{array}{l}15-35 \% \\
35-60 \% \\
60-90 \% \\
\end{array}\end{array}$ & $\begin{array}{l}60-35 \\
50-30 \\
40-25 \\
25-20\end{array}$ & $\begin{array}{l}85-60 \\
75-55 \\
65-50 \\
50-45\end{array}$ & $\begin{array}{l}90-65 \\
80-60 \\
70-55 \\
55-50\end{array}$ & $\begin{array}{l}\text { Groundnuts, sesame, watermelon, } \\
\text { potato, olives, citrus, mango. }\end{array}$ \\
\hline
\end{tabular}


Table 5. Cont.

\begin{tabular}{|c|c|c|c|c|}
\hline \multicolumn{5}{|c|}{$\begin{array}{l}\text { Factor (a) : More stable soil profile characteristics } \\
\text { Soil characteristics }\end{array}$} \\
\hline Soil characteristics & $\begin{array}{l}\text { Surface } \\
\text { irrigation }\end{array}$ & $\begin{array}{l}\text { Sprinkler } \\
\text { or drip } \\
\text { irrigation }\end{array}$ & & Specific crops \\
\hline 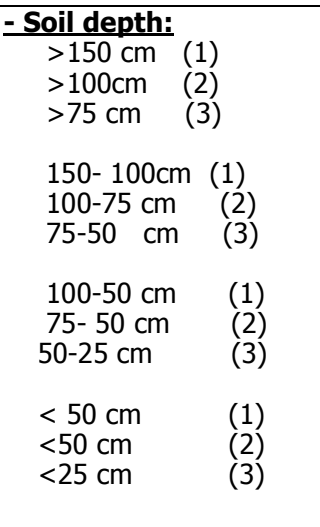 & & 95 & $100-90$ & $\begin{array}{l}\text { Cabbage, potato, cowpea, } \\
\text { pea, beans, onion, sorghum, } \\
\text { barley, wheat, maize, } \\
\text { groundnuts, sesame, guava, } \\
\text { grape, banana. } \\
\text { Cabbage, cowpea, sorghum, } \\
\text { barley, wheat, potato, onion, } \\
\text { guava. } \\
\text { Sorghum, cabbage, barley, } \\
\text { wheat, onion, guava. }\end{array}$ \\
\hline \multicolumn{5}{|c|}{ (1) Rock or hardpan. (2) $>50 \%$ lime or $>40 \%$ gypsum. (3) $>90 \%$ gravel } \\
\hline$\frac{\text { Lime content: }}{1-15 \%}$ & \multicolumn{2}{|l|}{$100-95$} & \multirow{3}{*}{$100-90$} & \multirow{3}{*}{$\begin{array}{l}\text { Olives, grape, barley, wheat, } \\
\text { sorghum, groundnuts. }\end{array}$} \\
\hline $\begin{array}{l}<1 \% \\
15-35 \%\end{array}$ & \multicolumn{2}{|l|}{$95-85$} & & \\
\hline $35-50 \%$ & \multicolumn{2}{|l|}{$85-75$} & & \\
\hline$\frac{\text { Gypsum content: }}{<1 \%}$ & 95 & 95 & 100 & \multirow{3}{*}{$\begin{array}{l}\text { Beans, banana, carrot, citrus, } \\
\text { green pepper, mango, onion, } \\
\text { soya, tomato. }\end{array}$} \\
\hline $\begin{array}{c}1-10 \% \\
10-15 \%\end{array}$ & $\begin{array}{l}100 \\
95\end{array}$ & 100 & $\frac{100-80}{80-60}$ & \\
\hline $15-40 \%$ & $95-50$ & $100-80$ & $60-40$ & \\
\hline \multicolumn{5}{|c|}{ Factor (b): Relatively less stable characteristics. } \\
\hline Soil characteristics & $\begin{array}{l}\text { Surface } \\
\text { irrigation }\end{array}$ & $\begin{array}{l}\text { Sprinkler } \\
\text { or drip } \\
\text { irrigation }\end{array}$ & $\begin{array}{l}\text { Perfect } \\
\text { drainage } \\
\text { system }\end{array}$ & Specific crops \\
\hline $\begin{array}{l}\text {-Wetness: } \\
\text { Well drained. } \\
\text { Water table }>150 \mathrm{~cm} ; \\
\text { Permeability }>6 \mathrm{~cm} / \mathrm{h}\end{array}$ & $100-90$ & \multirow[t]{2}{*}{$100-90$} & \multirow{3}{*}{$100-85$} & \multirow{3}{*}{$100-90$} \\
\hline $\begin{array}{l}\text { Moderately Well drained. } \\
\text { Water table } 150-100 \\
\mathrm{~cm} ; \\
\text { Permeability } 6-2 \mathrm{~cm} / \mathrm{h}\end{array}$ & $90-70$ & & & \\
\hline $\begin{array}{l}\text { Imperfectly drained. } \\
\text { Water table } 100-50 \mathrm{~cm} ; \\
\text { Permeability 2-0.6 } \\
\mathrm{cm} / \mathrm{h}\end{array}$ & $70-40$ & $90-75$ & & \\
\hline $\begin{array}{l}\text { Poorly drained. } \\
\text { Water table }<50 \mathrm{~cm} ; \\
\text { Permeability }<0.6 \mathrm{~cm} / \mathrm{h}\end{array}$ & $40-15$ & $75-55$ & $85-65$ & $90-75$ \\
\hline
\end{tabular}


Table 5. Cont.

Factor (b): Relatively less stable characteristics.

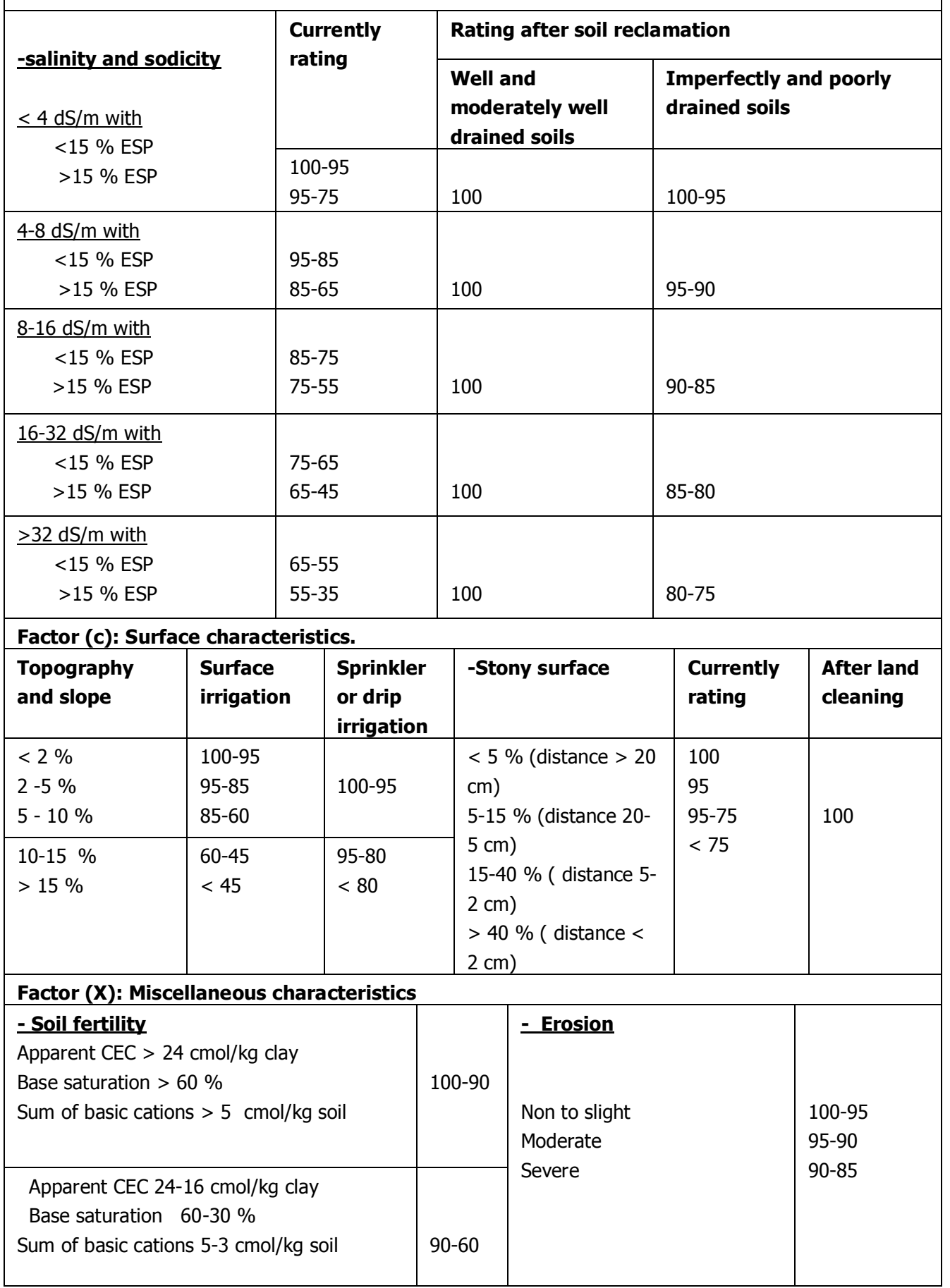


- The guidelines for soil profile description and the criterias of soil taxonomic units in both American and FAO systems were used as limits of soil characteristics rating. For example, the $15-40 \%$ as many stony surface; $5-10 \%$ as undulating topography; texture classes were grouped under the soil family names; more than $35 \%$ coarse fragments as very gravelly and skeletal particle-size class; $15 \%$ or more gypsum as gypsic mineralogy class and gypsic horizon according to FAO (1998) system; $40 \%$ or more gypsum and more than $90 \%$ coarse fragments as gypseous and fragmental substitutes particle-size classes, respectively; $15 \%$ or more and $50 \%$ or more lime as calcic and hypercalcic horizons, respectively.

- The weighted average of soil characteristics were evaluated to a depth of 1.5 meter for texture and to 1-m. for lime, gypsum and salinity contents. The soil profiles with stratified or heterogeneous layers were subdivided to the surface $0-25 \mathrm{~cm}, 25-50$ and $50-100 \mathrm{~cm}$ as soil control section, and $100-150 \mathrm{~cm}$ as substratum layers. The weighted average of these 4 sections is recalculated according to soil profile depth as the following:

$\begin{array}{lcccc}>100 \mathrm{~cm} & 0-25 \times 1.75, & 25-50 \times 1.25, & 50-100 \times 1, & 100-150 \times 0.5, \\ & (43.75) & (31.25) & (50) & (25) \\ 50-100 \mathrm{~cm} & 0-25 \times 1.5, & 25-50 \times 1, & 50-100 \times 0.75, \\ & (37.5) & (25) & (37.5) \\ 25-50 \mathrm{~cm} & 0-25 \times 1.25, & 25-50 \times 0.75, \\ & (31.25) & (18.75) \\ 0-25 \mathrm{~cm} & 0-25 \times 1, & \end{array}$

(25)

- The suitable rates were chosen within the rating range in the table that arranged descendingly for each soil characteristics. For example, the rating scale of the sandy family class with less than $15 \%$ c.f. ranges from 60 - $35 \%$ were arranged desendingly for LfS, LS, LCS, fS, S and CS texture classes. The highest rate refers to LfS while the lowest one is for CS and the other classes are between by its arrangement. Also, the $60 \%$ rate of LfS decreases gradually by increasing gravel content and reaches to $50 \%$ that refers to the highest rate of the some texture with $15-35 \%$ c.f.

- The rating of soil characteristics within each factor were multiplied together and the Storie Land Index is used to obtain the overall index by multiplying factor (a) $\mathbf{x}$ factor (b) / $100 \times$ factor (c) / $100 \times$ factor (x) / 100 . 
Six suitability classes were proposed having suitability indices $(\mathrm{Si})$ as follows:
$\mathrm{S} 1(\mathrm{Si} \geq 80)$ Highly Suitable
S4 (20 - 39) Marginally Suitable
S2 (60 - 79) Suitable
SC $(10-19)$ Conditionally Suitable

S3 (40 - 59) Moderately SuitableN $(\mathrm{Si}<10)$ Unsuitable

The conditionally Suitable class (SC) is considered as the special use class in the USBR scheme, or by definition in the FAO Framework (1976) as a phase of land suitability order Suitable, employed in circumstances where small areas of land within the survey are poorly suitable for a particular use under the management specified for that use.

- The proposed system is listed in one chart to serve-in some extend-the qualitative land suitability concerned with the objectives of the evaluation. The chose of rating depend upon the current or potential suitability as well as the level of intensity of the survey.

\section{Application of the proposed rating:}

By using the proposed rating, the obtained current land suitability of the investigated soil profiles are shown in Table (6). The suitability classes refer to its present condition as virgin soils (10 sites) and cultivated (12 sites) either with existing or improved management practices. The results indicate the following:

- Suitability classes of the cultivated surface irrigation soils ( 9 sites) are almost similar to that obtained by the USBR quantitative base (Griffiths, 1975) and potential Storie (1978) methods. The less suitability grade were observed only in the recently reclaimed soils (site 7) and also in the insufficient soil drainage system (site 19). For comparison, the Sys methods which assessment under the same soil factors indicate only 3 or 2 out of these 9 sites similar to USBR and Storie methods.

- Only one site indicates unsuitable class which in agreement with the USBR and Storie methods, while most of the investigated other methods indicate 4 to 7 unsuitable class. Also, the proposed rating correct in some extent the differences between the Sys methods in most the investigated sites (Table 7). It is more closer to the average of the three Sys methods with exception the Recent Nile alluvial and windblown sand soil sites which indicate more high indices. These results are more reliable under the conditions prevailing in the soils of Egypt.

- According to the structure of the FAO Framework (1976), the symbols of both suitability subclasses and units are shown in Table (6). It reflects kinds of limitations and minor aspect of their management requirements. The using of such symbol depends upon the level of survey study and the objectives of land evaluation.

In conclusion, such data can be used for a qualitative evaluation in the preproject, reconnaissance and even detailed phase of the survey. It will further help to select the most suitable lands for certain irrigation,system for which the economic land evaluation has to be made during the feasibility study. Also, it should be mentioned that neither land use requirements nor specific crop requirement are 
absolute data, both may be modified by different systems of land management. Similarly, land conditions are often not completely fixed, but may be modified by systems of management, e.g. the application of water and/or fertilizers, or by methods of land improvements, e.g. leaching of salts, methods of drainage, deep plowing of soils and removal of hardpans.

Table 6. Suitability evaluation of the investigated soils by using the proposed rating

\begin{tabular}{|c|c|c|c|c|c|c|c|c|c|c|c|c|}
\hline \multirow{2}{*}{$\begin{array}{c}\text { Profile } \\
\text { No }\end{array}$} & \multicolumn{4}{|c|}{ Factor (a) } & \multicolumn{2}{|c|}{ Factor (b) } & \multicolumn{2}{|c|}{ Factor (c) } & \multicolumn{4}{|c|}{ Land suitability } \\
\hline & 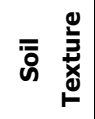 & $\overline{\bar{c}}$ & 嵩 & 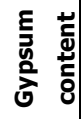 & $\begin{array}{l}y \\
\vdots \\
⿱ 亠 䒑 \\
\vdots\end{array}$ & 胥 & 혀 & $\begin{array}{l}\vec{d} \\
\text { जे } \\
\text { जै }\end{array}$ & 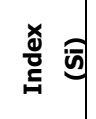 & $\begin{array}{l}\text { y } \\
\frac{\pi}{U}\end{array}$ & जे 气ू & 蒙 \\
\hline \multicolumn{13}{|c|}{ Recent Nile alluvial soils (Nile Delta) } \\
\hline 1 & 83.0 & 100 & 100 & 100 & 90 & 100 & 100 & 100 & 74.7 & S2 & S2a & S2a-1 \\
\hline 2 & 78.5 & 100 & 100 & 100 & 80 & 95 & 100 & 100 & 59.7 & S2/S3 & S2/S3ab & S2/S3ab-1 \\
\hline 3 & 92.0 & 100 & 100 & 98 & 100 & 100 & 100 & 100 & 90.2 & S1 & - & - \\
\hline 4 & 80.4 & 100 & 100 & 100 & 100 & 100 & 100 & 100 & 80.4 & $\mathrm{~S} 1$ & - & - \\
\hline \multicolumn{13}{|c|}{ Fluvio-Marine soils } \\
\hline 5 & 82.3 & 100 & 100 & 100 & 63.0 & 80.0 & 100 & 100 & 41.5 & S3 & S3ab & S3ab-2 \\
\hline 6 & 85.0 & 100 & 100 & 100 & 65.0 & 85.0 & 100 & 100 & 47.0 & S3 & S3ab & S3ab-2 \\
\hline 7 & 78.2 & 100 & 100 & 95 & 50.0 & 70.0 & 100 & 100 & 27.4 & S4 & S4ab & S4ab-1 \\
\hline \multicolumn{13}{|c|}{ Old alluvial soils } \\
\hline 8 & 52.5 & 100 & 97 & 95 & 100 & 97 & 97 & 100 & 45.5 & S3 & S3a & S3a-1 \\
\hline 9 & 40.3 & 100 & 90 & 95 & 100 & 80 & 82 & 100 & 22.6 & S4 & S4abc & - \\
\hline 10 & 79.3 & 100 & 100 & 95 & 100 & 100 & 100 & 100 & 75.1 & S2 & $\mathrm{S} 2 \mathrm{a}$ & S2a-2 \\
\hline 11 & 51.1 & 100 & 97 & 95 & 100 & 65 & 97 & 100 & 29.7 & S4 & S4ab & S4ab-2 \\
\hline 12 & 64.7 & 100 & 95 & 100 & 92 & 70 & 90 & 100 & 35.6 & S4 & S4ab & S4ab-3 \\
\hline 13 & 76.8 & 100 & 100 & 95 & 90 & 83 & 95 & 100 & 51.8 & S3 & S3ab & S3ab-3 \\
\hline 14 & 55.0 & 93 & 90 & 100 & 90 & 80 & 90 & 100 & 29.8 & S4 & S4ab & S4ab-3 \\
\hline \multicolumn{13}{|c|}{ Windblown sand soils } \\
\hline 15 & 39.0 & 100 & 100 & 95 & 100 & 100 & 100 & 100 & 37.1 & S4 & S4a & - \\
\hline 16 & 67.0 & 100 & 96 & 95 & 100 & 97 & 100 & 100 & 59.3 & $\mathrm{~S} 2 / \mathrm{S} 3$ & $\mathrm{~S} 2 / \mathrm{S} 3 \mathrm{a}$ & S2/S3a-2 \\
\hline \multicolumn{13}{|c|}{ Coastal plain soils } \\
\hline 17 & 41.0 & 100 & 95 & 95 & 48 & 50 & 100 & 100 & 8.9 & $\mathrm{~N}$ & $\mathrm{Nab}$ & - \\
\hline 18 & 90.6 & 100 & 93 & 100 & 100 & 100 & 100 & 100 & 84.3 & S1 & - & - \\
\hline 19 & 72.7 & 100 & 90 & 100 & 70 & 97 & 100 & 100 & 44.4 & S3 & S3ab & S3ab-4 \\
\hline 20 & 80.7 & 100 & 85 & 100 & 95 & 85 & 90 & 100 & 49.9 & S3 & S3ab & S3ab-4 \\
\hline \multicolumn{13}{|c|}{ Miscellaneous land types } \\
\hline 21 & 75.4 & 65 & 100 & 100 & 60 & 77 & 95 & 100 & 21.2 & S4 & S4ab & S4ab-4 \\
\hline 22 & 57.8 & 60 & 100 & 100 & 70 & 55 & 90 & 100 & 12.0 & SC & SCab & - \\
\hline
\end{tabular}

S1 Highly suitable ( $\mathrm{Si} \geq 80$ )

S2 Suitable (Si 60-79)

S3 Moderately suitable (Si 40-59)
S4 Marginally suitable (Si 20-39)

SC Conditionally suitable (Si 10-19)

$\mathrm{N} \quad$ Unsuitable $(\mathrm{Si}<10)$ 
Table 7. The relation between the proposed suitability index and the indices of Sys methods

\begin{tabular}{|c|c|c|c|c|c|}
\hline Profile No.* & $\begin{array}{c}\text { Average of } \\
\text { Sys methods }\end{array}$ & 1974 & 1978 & 1991 & $\begin{array}{c}\text { Proposed } \\
\text { index } \\
\end{array}$ \\
\hline \multicolumn{6}{|c|}{ Recent Nile alluvial soils } \\
\hline 1 & 69.9 & +6.1 & +0.8 & -7.0 & +4.8 \\
\hline 2 & 52.3 & +7.6 & +0.3 & -7.8 & +7.4 \\
\hline 3 & 88.5 & +6.5 & -2.2 & -4.3 & +1.7 \\
\hline 4 & 68.8 & +2.5 & +6.2 & -8.8 & +11.6 \\
\hline Average & 69.9 & +5.7 & +1.3 & -7.0 & +6.35 \\
\hline \multicolumn{6}{|c|}{ Fluvio-Marine soils } \\
\hline 5 & 41.9 & -2.5 & +6.6 & -4.0 & -0.4 \\
\hline 6 & 42.5 & +2.7 & +3.5 & -6.2 & +4.5 \\
\hline 7 & 27.4 & -0.1 & +4.7 & -4.5 & 0.0 \\
\hline Average & 37.3 & 0.0 & +4.9 & -4.9 & +1.3 \\
\hline \multicolumn{6}{|c|}{ Old alluvial plain soils } \\
\hline 8 & 41.5 & +5.7 & +0.9 & -6.7 & +4.0 \\
\hline 9 & 21.8 & +7.0 & +3.2 & -10.2 & +0.8 \\
\hline 11 & 31.2 & +5.6 & +1.4 & -6.9 & -1.5 \\
\hline 12 & 42.0 & +9.8 & +8.0 & -17.8 & -6.4 \\
\hline 13 & 52.4 & +9.0 & +4.8 & -13.7 & -0.6 \\
\hline 14 & 27.3 & +6.7 & +1.0 & -8.2 & +2.5 \\
\hline Average & 36.0 & +7.4 & +3.3 & -10.6 & -0.17 \\
\hline \multicolumn{6}{|c|}{ Windblown sand soils } \\
\hline 15 & 26.1 & -0.4 & -0.4 & +0.9 & +11 \\
\hline \multicolumn{6}{|c|}{ Coastal plain soils } \\
\hline 17 & 8.2 & -0.2 & -0.2 & +0.3 & +0.7 \\
\hline 19 & 51.5 & +6.6 & +2.1 & -8.6 & -7.1 \\
\hline 20 & 49.1 & +8.4 & +6.3 & -14.8 & +0.8 \\
\hline Average & 36.2 & +5.0 & +2.8 & -7.6 & -1.8 \\
\hline \multicolumn{6}{|c|}{ Miscellaneous land types } \\
\hline 21 & 31.3 & -1.3 & +0.9 & +0.3 & -10.1 \\
\hline 22 & 14.3 & +3.7 & +1.7 & -5.4 & -2.3 \\
\hline Average & 22.3 & +1.2 & +1.3 & -2.6 & -6.2 \\
\hline
\end{tabular}

*Without the sprinkler and drip irrigation sites. 


\section{REFERENCES}

1. Beek, K. J. 1978. Land evaluation for agricultural development. Publication No. 23, International Institute for Land Reclamation and Improvement, Wageningen, the Netherlands.

2. Bowser, W. E. and H. C. Moss. 1950. A soil rating and classification for irrigation lands in western Canada, Scientific Agriculture 30: 165-171.

3. Brinkman, R. and A. J. Smyth (eds). 1973. Land evaluation for rural purposes. Publication No. 17, International Institute for Land Reclamation and Improvement, Wageningen, the Netherlands.

4. Clarke, G. R. 1951. The evaluation of soils and the definition of quality class from studies of the physical properties of the soil profile in the field. Journal of Soil Science 2, 50-60.

5. El-Nahal, M. A., R. M. Abd El-Aal, A. A. Abd El-Wahed and I. Raafat. 1977. Soil studies on the Nile Delta. Egypt. J. Soil Sci., 17, No. 1, 55-65.

6. FAO. 1976. A Framework for Land Evaluation. Soil Bull. No. 32, Rome, Italy.

7. FAO. 1979. Land evaluation criteria for irrigation. Report of an Expert consultation, 27 February- 2 March 1979. World Soil Resources. Report No. 50, FAO Rome, 219.

8. FAO. 1990. Guidelines for Soil Description. $3^{\text {rd }}$ Edition, SRMCS, LWDD, FAO, Rome, Italy.

9. FAO. 1998. World Reference Base for Soil Resources. World Soil Resources Reports 84, FAO, Rome.

10. Griffiths, E. 1975. Classification of land for irrigation in New Zealand. New Zealand Soil Bureau, Scientific Report No. 22, Department of Scientific and Industrial Research, New Zealand.

11. Nelson, L. A. 1963. Detailed land classification. Island of Oahu. Bull. Ld. Study Bur. Univ. Hawaii No. 3.

12. Olson, G. W. 1974. Land classification. Search, Agriculture 4, 1 - 34.

13. Smith, R. T. and K. Atkinson. 1975. Techniques in pedology. Elek Science, London.

14. Storie, R. E. 1954. Land classification as used in California for appraisal of land for taxation purposes. Trans. $5^{\text {th }}$ Int. Cong. Soil Sci., 3: 407-420.

15. Storie, R. E. 1978. Storie index rating. Oakland, University of California Division of Agricultural Sciences, Special Publication 3203.

16. Sys, C. 1980. Land evaluation. International Training Center for Post-Gradute Soil Scientists, State University of Ghent, Belgium. 
17. Sys, C. and W. Verheye 1972. Principles of land classification in arid and semiarid regions. I.T.C., Ghent, Belgium.

18. Sys, C. and W. Verheye 1974. Land evaluation for irrigation of arid regions by the use of the parameter. Method. Transaction $10^{\text {th }}$ International Congress of Soil Science V, 149-155.

19. Sys, C. and W. Verheye. 1978. An attempt to the evaluation of physical land characteristics for irrigation according to the FAO Framework for Land Evaluation. International Training Center for Post-Gradute Soil Scientists, State University of Ghent, Belgium.

20. Sys, C., E. Van Ranst and J. Debaveye. 1991. Land Evaluation. Part I and II, International Training Center for Post-Gradute Soil Scientists, State University of Ghent, Belgium. Agric. Public. No. 7. General Adminstration For Development Cooperation, Brusseis, Belgium.

21. Sys, C., E. Van Ranst, J. Debaveye and F. Beernaert 1993. Land Evaluation. Part III: Crop Requirements. Agric. Public. No. 7, General Adminstration For Development Cooperation, Brassels, Belgium.

22. USBR. 1953. Reclamation Manual. Vol. V: Irrigated Land Use, Part 2: Land Classification. United States Bureau of Reclamation, Denver, Colorado, USA.

23. USDA. 1993. Soil Survey Manual. USDA Handbook No. 18, U.S. Government Printing Office, Washington, D.C., USA.

24. USDA. 2004. Soil Survey Laboratory Methods Manual. Soil Survey Investigation Report, No. 42, Version 4 November, Washington, USA.

25. USDA. 2014. Keys to Soil Taxonomy. $12^{\text {th }}$ Edition, USDA.

26. Veenenbos, C.A. 1963. High Dam soil survey. FAO/SF: 16 UAR.

27. Vink, A. P. A. 1975. Land use in advancing agriculture Springer-Verlag, Berlin.

28. Zonn, I. 1977. Irrigation of the world's arid lands. World Crops and Livestock 29, 72-73. 


\title{
نموذج معدل لتقييم صلاحية الأراضى للزراعة المروية
}

\author{
ابرهيم فوزى رشاد \\ معر بحوث الأراضى والمباه والبيئة - مركز البحوث الزراعية - الجبزة، مصر
}

التعديلات المقترحة لتقديرات صفات التربة تم إجر اءها بدراسة مقارنة بين الطرق المستخدمة

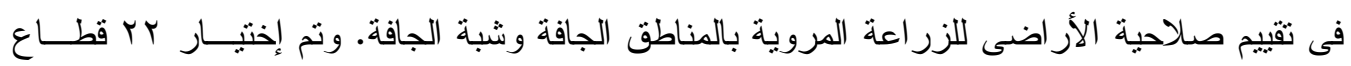

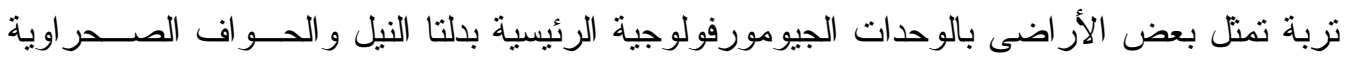

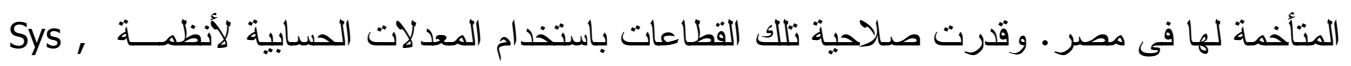

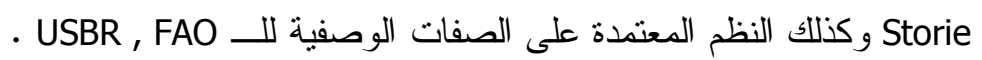

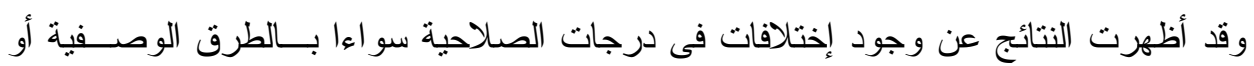

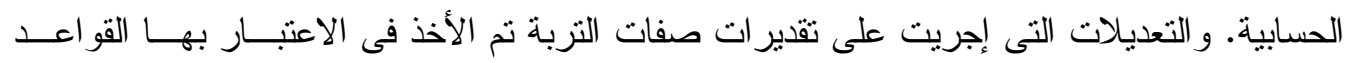

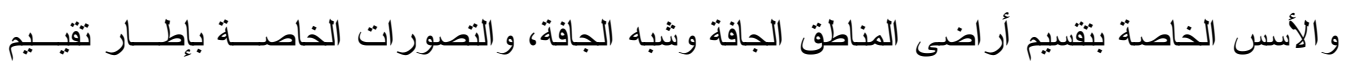
الأر اضى للـ FAO ، وطرق Sys, Storie ، وكذللك على حدود أدلة وصف قطاعات التربة ومعايير

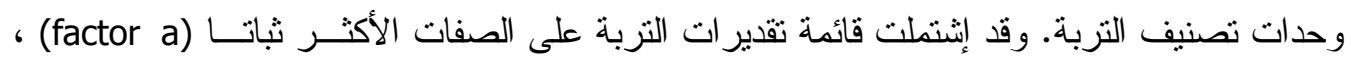

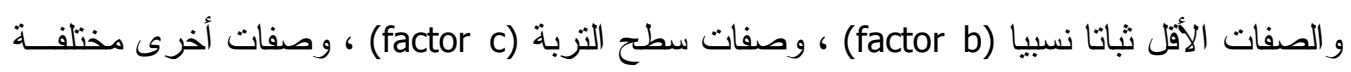
(factor x)

\section{Si = factor (a) $x$ factor (b) / $100 \times$ factor (c) / $100 \times$ factor (x) / 100}

S4 S4 - 20) Sامشية الصلاحية

(19) - 10) SC

(10 > Si) N غير صالحة

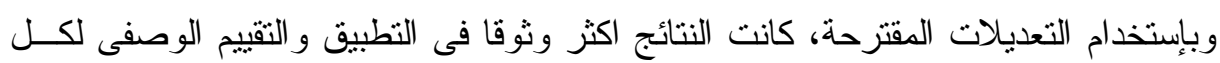

من درجات الصلاحية الحالية والمتوقعة لتتاسب الدر اسات الاولية و الاستكثافية وكذلك الأكثر تفصيلا 